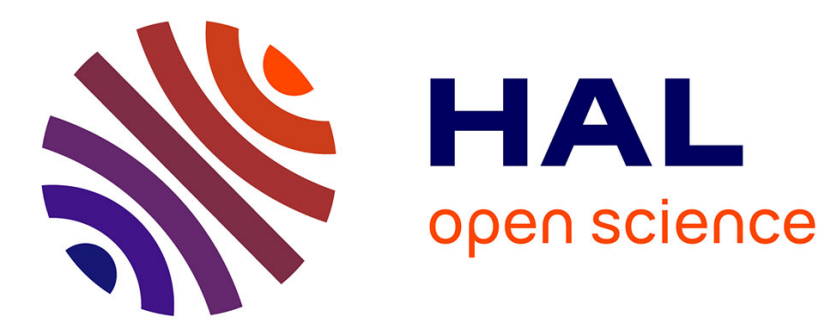

\title{
Compact and fault-tolerant photonic crystal add drop filter
}

\author{
Ségolène Olivier, Claude Weisbuch, Henri Benisty
}

\section{To cite this version:}

Ségolène Olivier, Claude Weisbuch, Henri Benisty. Compact and fault-tolerant photonic crystal add drop filter. Optics Letters, 2003, 28 (22), pp.2246-2248. 10.1364/OL.28.002246 . hal-00878523

\section{HAL Id: hal-00878523 \\ https://hal-iogs.archives-ouvertes.fr/hal-00878523}

Submitted on 30 Oct 2013

HAL is a multi-disciplinary open access archive for the deposit and dissemination of scientific research documents, whether they are published or not. The documents may come from teaching and research institutions in France or abroad, or from public or private research centers.
L'archive ouverte pluridisciplinaire HAL, est destinée au dépôt et à la diffusion de documents scientifiques de niveau recherche, publiés ou non, émanant des établissements d'enseignement et de recherche français ou étrangers, des laboratoires publics ou privés. 


\title{
Compact and fault-tolerant photonic crystal add-drop filter
}

\author{
S. Olivier and C. Weisbuch \\ Laboratoire de Physique de la Matière Condensée, Ecole Polytechnique, 91128 Palaiseau, France
}

\author{
H. Benisty \\ Laboratoire de Physique de la Matière Condensée, Ecole Polytechnique, 91128 Palaiseau, France, and \\ Laboratoire Charles Fabry, Institut d'Optique, 91403 Orsay, France
}

Received April 25, 2003

\begin{abstract}
We propose an add-drop filter consisting of two adjacent waveguides carved into a two-dimensional photonic crystal that is etched through a standard guiding structure. This filter is based on distributed energy transfer via the frequency-selective intermediate conversion of the fundamental guided mode to a high-order low-group-velocity mode. This geometry circumvents the fabrication sensitivity on the single-hole scale of previous cavity-based designs. Combining distributed energy transfer and reduced group velocity preserves compactness. The design is analytically optimized with a coupled-mode approach. ( 2003 Optical Society of America

OCIS codes: $130.0130,230.7380$.
\end{abstract}

In a multimode waveguide formed by two close reflective surfaces, if a corrugation of period $a$ is created, waveguiding and diffraction compete. Guiding is frustrated when a Bragg condition $\Delta \mathbf{k}_{\|}=m(2 \pi / a)$ is met between the wave vectors $\mathbf{k}_{\|}$of any two modes. This is the situation in photonic crystal channel waveguides (PCCWs) that are defined by a few missing rows in a two-dimensional photonic crystal, e.g., an array of holes perforating a dielectric slab guide, sketched in Fig. 1(a), between the fundamental mode and a higher-order mode. A mode gap arises in the dispersion diagram as well as a transmission mini-stop-band (MSB), a feature that is also well documented for lossy modes. ${ }^{1-3}$

For the miniature add-drop filters desired for optical networks the use of tiny cavities coupled to a PCCW shows promise ${ }^{4-6}$ but presents severe difficulties. For single-hole defects ${ }^{5,6}$ even a few nanometers on the diameter of nearby holes shift the cavity mode and influence its coupling. Posttrimming is required to fabricate devices in deterministic channels. This hindrance does not exist for distributed-feedback lasers because the feedback is averaged over several hundred periods of $a \sim \lambda / 2 n$ ( $\lambda$ is the wavelength, and $n$ is an effective index). However, for multiple wave interferences, scattering from a few tens of scatterers results in a huge coherent intensity compared with out-of-phase directions. ${ }^{7}$ This suggests that the distribution of a high-performance filtering action is compatible with a core device size of the order of $10-20 \lambda / n \sim 10 \mu \mathrm{m}$.

Our add-drop proposal exploits the MSB of two adjacent PCCWs [Fig. 1(c)] in the quasi-TE polarization of the photonic bandgap. In its first form it steers light from the top PCCW fundamental mode a into the high-order, low-group-velocity mode $b$ at the specific MSB frequency. In this new state, light tunnels to the adjacent high-order mode $b$, where the reciprocal MSB effect retrieves mode a directionally. The large transverse momentum of the two $b$ modes and their far deeper penetration in the barrier [see the magnetic field map of both modes in Fig. 1(b)] greatly simplify the design, allowing the codirectional transfer of the a modes in the $\sim 10 \mu \mathrm{m}$ of our system to be fully neglected; the coupling length reaches $\sim 1 \mathrm{~mm}$ for a separation of a few rows. Figure 1(d) provides a heuristic quantum-mechanical analogy. The implementation of an extra transverse cavity to sharpen filtering is illustrated in Figs. 1(e) and 1(f). The concept is fault tolerant since no wavelet or localized mode is singled out. The PCCW assists the $a-b$ and $b-a$ conversions while ensuring compactness through the low group velocity of the $b$ modes. These highly confined modes take advantage of the light-insulating property of the photonic crystal. Similar modes would be very leaky in ridge channel waveguides with some corrugation. For integration in a device the four access guides can be of the ridge type or PCCWs. Their spacing prevents codirectional coupling, while a PCCW access guide with a slightly detuned period or width can be used to eliminate the MSB phenomenon in the desired frequency range.

Applying coupled-mode theory (CMT) to obtain an analytical optimization of the waveguide parameters (width, length, spacing) allows for efficient design. Use of CMT has recently been validated for the MSB of a single PCCW on data from a GaAs-based PCCW, ${ }^{3}$ yielding knowledge of even the modal losses $\alpha_{a}$ and $\alpha_{b}$. The other components are the group velocities $n_{g a}$ and $n_{g b}$ of the two modes and their coupling constant $\kappa_{a b}$. These quantities are obtained numerically from the slopes of the mode dispersion and the mode spectral gap, which can be expressed as $\Delta u_{a b}=\left(2 a \kappa_{a b}\right) /\left[\pi\left(n_{g a}+n_{g b}\right)\right]$, where $u=a / \lambda$ is the normalized frequency. Adjusting $\alpha_{a}$ and $\alpha_{b}$ could yield a good fit to the experimental transmission data from GaAs-based PCCWs of lengths from $30 a$ to $240 a{ }^{3}$

The filter in Fig. 1(c) can be simply modeled by the following coupled-mode equations between the amplitudes $A_{1}, A_{2}, B_{1}$, and $B_{2}$ of the fundamental $\mathrm{a}$ and high-order $\mathrm{b}$ modes in the upper (subscript 1 ) 


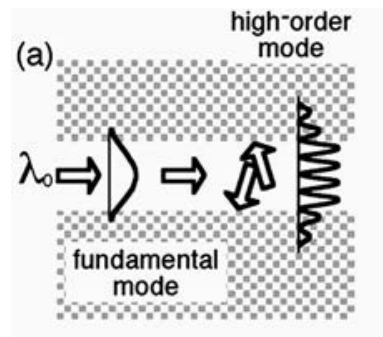

(c)

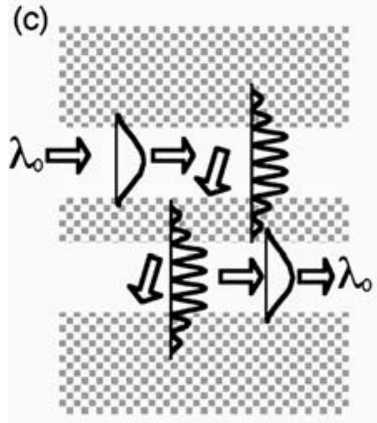

(e)

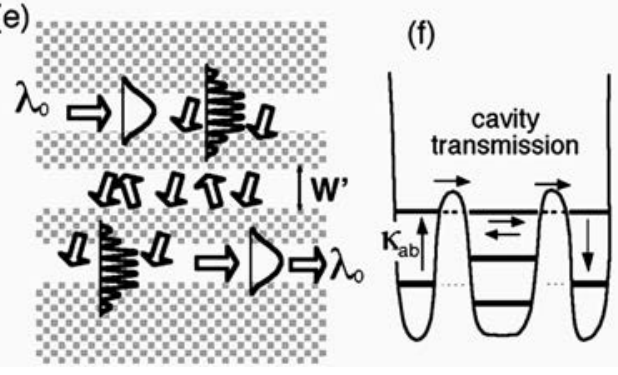

Fig. 1. (a) Mode coupling from the fundamental mode a to a high-order mode $b$ in a photonic crystal waveguide. (b) Map of the magnetic field amplitude of fundamental and higher-order TE modes in a particular waveguide with four missing rows [see Fig. 2(a)]. (c) Add-drop filter based on the $a-b$, tunneling of the $b$ modes, and $b-a$ mode conversions. (d) Picture with two wells showing the easier tunneling of the higher-order $\mathrm{b}$ modes. (e) Insertion of $\mathrm{a}$ Fabry-Perot-type cavity of modest width $W^{\prime}$ to sharpen filtering. (f) Picture of three wells showing the filtering operation.

and lower (subscript 2) waveguides, with obvious notations ${ }^{8}$ :

$$
\frac{\mathrm{d}}{\mathrm{d} z}\left(\begin{array}{c}
A_{1} \\
B_{1} \\
A_{2} \\
B_{2}
\end{array}\right)=\left(\begin{array}{cccc}
-i \delta_{a} & -i \kappa_{a b} & 0 & 0 \\
i \kappa_{a b} & i \delta_{b} & 0 & i \kappa_{b b} \\
0 & 0 & -i \delta_{a} & -i \kappa_{a b} \\
0 & i \kappa_{b b} & i \kappa_{a b} & i \delta_{b}
\end{array}\right)\left(\begin{array}{c}
A_{1} \\
B_{1} \\
A_{2} \\
B_{2}
\end{array}\right)
$$

where $\kappa_{b b}$ describes the codirectional coupling between the higher-order b modes, whereas $\kappa_{a a}$ is simply neglected. $\delta_{a}$ and $\delta_{b}$ are the detuning with respect to the Bragg condition.

We find the optimal design analytically in the lossless regime, while ordinary mathematics reveals the evolution of the performance with these optimal parameters for the lossy case. The output waves are calculated for $A_{1}(0)=1, B_{1}(L)=0, A_{2}(0)=0$, and $B_{2}(L)=0$; i.e., only mode a is launched into channel 1. To obtain optimal conditions, we impose unity amplitude of the fundamental mode in the cross port $\left[A_{2}\left(L_{\text {opt }}\right)=1\right]$ and zero in the bar port $\left[A_{1}\left(L_{\text {opt }}\right)=0\right]$. We find this critical coupling when

$$
\kappa_{a b}=\frac{\sqrt{5}}{6} \kappa_{b b}, \quad L_{\mathrm{opt}}=\pi\left(\frac{\kappa_{b b}^{2}}{4}-\kappa_{a b}^{2}\right)^{-1 / 2}=\frac{3 \pi}{\kappa_{b b}}
$$

Because mode b goes backward, we find that $A(z)$ and $B(z)$ exhibit nonmonotonic behavior along the waveguides at criticality, notably with $B_{1}\left(L_{\text {opt }} / 3\right)=0$.

We now present a specific example based on PCCWs with four missing rows with symmetric boundaries (which we label W4) and made of a triangular array of air holes etched through an InP-based heterostructure (with an air-filling factor of $f=0.35$ and a background dielectric constant of $\epsilon=10.4$ ). Figure 2(a) shows an enlargement of the dispersion relations of an isolated W4 waveguide with symmetric boundaries computed by the plane-wave method. ${ }^{9}$ Figure 1(b) illustrates the modes at $k=0.07 \pi / a$. A minigap of width $\Delta u_{a b}=1.14 \times 10^{-3}$ between the fundamental mode $\left(n_{g a}=3.28\right)$ and high-order mode $7\left(n_{g b}=71\right)$ occurs at the normalized frequency $u_{0}=a / \lambda=0.282$, leading to a dimensionless coupling constant $a \kappa_{a b}=$ 0.133. Then Eq. (2) dictates $a \kappa_{b b}=0.357$ for optimal operation. A close value, $a \kappa_{b b}=0.361$, is obtained for a separation of four rows, which is related to a splitting of mode b of $\Delta u_{b b}=1.62 \times 10^{-3}$ determined far from the MSB region. The optimal length for full transfer (cross operation) is then $L_{\mathrm{opt}}=26 a$, yielding $11 \mu \mathrm{m}$ when $\lambda=1.5 \mu \mathrm{m}(a \sim 0.44 \mu \mathrm{m})$. Figure 3 shows the calculated bar and cross transmission spectra for the lossless case with the above parameters. The bar spectrum displays a sharp peak with a maximum at $98.7 \%$ and a quality factor of $Q=1500$. To filter out the unwanted sidelobes, a $26 a \times W^{\prime}$ cavity can be inserted between the two waveguides, as proposed in Fig. 1(e). From our knowledge of these

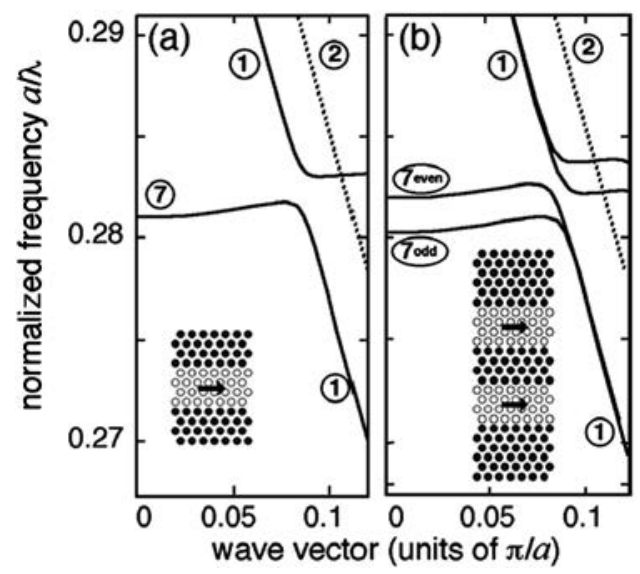

Fig. 2. (a) Dispersion relation of an isolated W4 PCCW with symmetric boundaries (shown in the inset) around the MSB between modes a (1) and $\mathrm{b}(7)$ at $u_{0}=0.282$ (the photonic crystal parameters are $f=0.35$ and $\epsilon=10.4)$. The modes in Fig. 1(b) are on branches 1 and 7 at $k=$ $0.07 \pi / a$. (b) Dispersion relation for guides separated by four rows (see inset) at the critical coupling condition. 


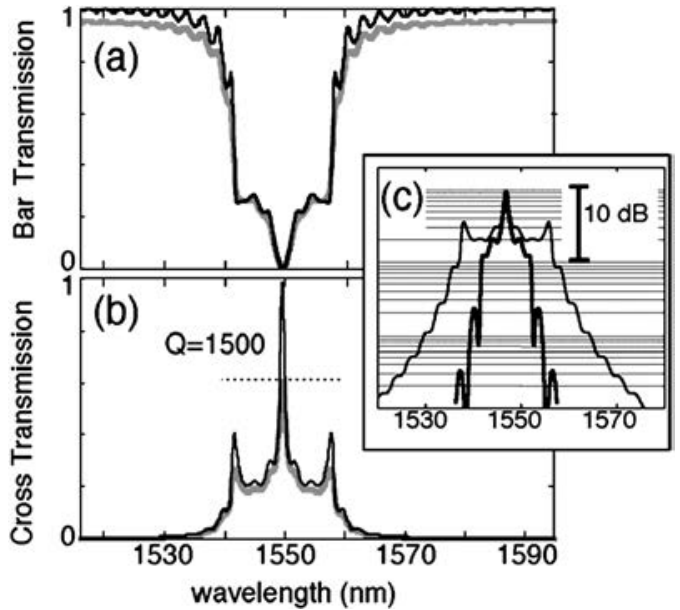

Fig. 3. (a) Bar channel transmission spectrum in the lossless case (black curve) and with propagation losses $\alpha_{a}=$ $20 \mathrm{~cm}^{-1}$ and $\alpha_{b}=400 \mathrm{~cm}^{-1}$ (gray curve). (b) Cross channel transmission spectrum in the same cases. (c) Semilog plot of the bar spectrum as in Fig. 1(b) (thin curve) and with the Fabry-Perot cavity filtering action (thick curve).

Fabry-Perot cavities, ${ }^{10}$ which we extrapolate to the latest trends toward low-loss planar photonic crystals, ${ }^{11,12}$ we infer a mirror reflectivity of $R=0.95$. With a physical cavity width $W^{\prime}$ of the order of $7 a$ to $8 a$ we have a dimensionless free spectral range of $\Delta \lambda / \lambda \sim$ 0.05 (the effective cavity length $W_{\text {eff }}$ is $9 a$ to $10 a$, and we assume $n_{g} \sim 7,2 n_{g} u \sim 2$ and use the basic relation $\left.\Delta \lambda / \lambda=\lambda / 2 n_{g} W_{\text {eff }}\right)$ and thus a quality factor $Q^{\prime} \sim 1220$ (with a cavity order of 20 and a finesse of 61 ). A simple but still realistic way to take its effect into account is to multiply $\kappa_{b b}$ in the coupled-mode calculation by the complex cavity transmission $T /[1-R \exp (i \varphi)]$, with $\varphi \equiv 2 k W^{\prime}$ being the classical cavity round-trip phase. As a rule, the product $\kappa_{b b} T /(1-R)$ should be kept constant. The resulting cross spectrum $[\mathrm{dB}]$ is shown in Fig. 3(c) as a thick curve. The overall $Q$ is slightly increased, whereas the former plateaus are rejected by 10 to $13 \mathrm{~dB}$. This is not the limit: The cavity configuration should be optimized independently.

Returning to the primary design [Figs. 1(c), 3(a), and $3(\mathrm{~b})]$, we checked that the bar signal remains below $5 \%(-13 \mathrm{~dB})$ for fluctuation of the air-filling factor $\delta f \sim \pm 0.01$ [in fact, $\delta\left(a \kappa_{b b}\right) \sim \pm 0.01$ ], for losses $\alpha_{b}$ as high as $\sim 1200 \mathrm{~cm}^{-1}$ (note that $n_{g b}=71$, however) and losses $\alpha_{a}$ as high as $100 \mathrm{~cm}^{-1}$ (far more than published in the case of a PCCW with three missing rows $\left.{ }^{13,14}\right)$. As an example, the gray curves in Figs. 3(a) and 3(b) are calculated for $\alpha_{a}=20 \mathrm{~cm}^{-1}$ and $\alpha_{b}=400 \mathrm{~cm}^{-1}$. In the first design proposal the insertion loss in the drop channel is as small as -1.5 to $-4 \mathrm{~dB}$ with the same large modal loss values [see the gray curve in Fig. 3(b)]. Finally, power that is dropped in the wrong direction takes the form of only a small amount of high-order-mode $B_{2}$ that does not sizably propagate back; i.e., the cross talk is negligible as in the case of $A_{2}(0)=0$. A detailed investigation of fault tolerance among holes is the next step and could be achieved by finite-difference time domain modeling, ${ }^{15}$ and a coupled-mode theory with a variable coupling constant could also provide direct insight.

In conclusion, our add-drop filter uses novel modecoupling phenomena specific to photonic crystal waveguides. Because of the use of distributed action and confined low-group-velocity modes, our filter shows acceptable performance for coarse wavelength division networks with the loss level established for state-of-the-art photonic crystal systems in two dimensions. ${ }^{11,12}$ In addition, it remains compact and makes genuine use of full in-plane confinement of photonic crystals.

This work was supported by the European Union's Information Society Technologies project 11239 PCIC on photonic crystal integrated circuits. ${ }^{16} \mathrm{H}$. Benisty's e-mail address is hb@pmc.polytechnique.fr.

\section{References}

1. S. Olivier, M. Rattier, H. Benisty, C. Weisbuch, C. J. M. Smith, R. M. De La Rue, T. F. Krauss, U. Oesterle, and R. Houdré, Phys. Rev. B 63, 113311 (2001).

2. S. Olivier, H. Benisty, C. J. M. Smith, M. Rattier, C. Weisbuch, and T. F. Krauss, Opt. Quantum Electron. 34, 171 (2002).

3. S. Olivier, H. Benisty, C. Weisbuch, C. J. M. Smith, and T. F. Krauss, Opt. Express 11, 1490 (2003), http:// www.opticsexpress.org.

4. C. J. M. Smith, R. M. De La Rue, M. Rattier, S. Olivier, H. Benisty, C. Weisbuch, T. F. Krauss, R. Houdré, and U. Oesterle, Appl. Phys. Lett. 78, 1487 (2001).

5. S. Noda, A. Chutinan, and M. Imada, Nature 407, 608 (2000).

6. S. Fan, P. R. Villeneuve, J. D. Joannopoulos, and H. A. Haus, Phys. Rev. Lett. 80, 960 (1996).

7. M. Born and E. Wolf, Principles of Optics (Pergamon, Oxford, 1970).

8. A. Yariv and P. Yeh, Optical Waves in Crystals (Wiley, New York, 1984).

9. H. Benisty, J. Appl. Phys. 79, 7483 (1996).

10. M. Rattier, H. Benisty, C. J. M. Smith, A. Béraud, D. Cassagne, T. F. Krauss, and C. Weisbuch, IEEE J. Quantum Electron. 37, 237 (2001).

11. M. Notomi, K. Yamada, A. Shinya, J. Takahashi, C. Takahashi, and I. Yokoyama, Phys. Rev. Lett. 87, 253902 (2001).

12. R. Ferrini, B. Lombardet, B. Wild, R. Houdré, S. Olivier, H. Benisty, A. Djoudi, L. Legouezigou, S. Hubert, S. Sainson, J.-P. Chandouineau, S. Fabre, F. Pommereau, and G.-H. Duan, Electron. Lett. 38, 962 (2002).

13. C. J. M. Smith, H. Benisty, S. Olivier, M. Rattier, C. Weisbuch, T. F. Krauss, R. M. De La Rue, R. Houdré, and U. Oesterle, Appl. Phys. Lett. 77, 2813 (2000).

14. E. Schwoob, H. Benisty, S. Olivier, C. Weisbuch, C. J. M. Smith, T. F. Krauss, R. Houdré, and U. Oesterle, J. Opt. Soc. Am. B 19, 2403 (2002).

15. M. Qiu, B. Jaskorzynska, M. Swillo, and H. Benisty, Microwave Opt. Technol. Lett. 34, 387 (2002).

16. http://www.intec.rug.ac.be/ist-optimist/ 